\title{
Stochastic Gust Analysis Techniques for Aircraft Conceptual Design
}

\author{
Krzysztof J. Fidkowski; $\quad$ Frode Engelsen† Karen E. Willcox $\ddagger$ Ilan M. Kroo§
}

\begin{abstract}
Dynamic loading constraints due to maneuver and gust loads are an important design consideration for novel aircraft configurations. Incorporating these constraints in the early design process with an appropriate level of fidelity presents a significant challenge, due both to the need for more detailed aerodynamics and control modeling, and to the stochastic nature of the gust encounter problem. This paper presents a stochastic gust analysis approach that is suitable for a multidisciplinary design optimization setting that incorporates dynamic loading constraints. The analysis approach employs Lyapunov and matched filter theory methods to address the stochastic nature of the gust response. A set of gust simulation studies are carried out to assess the relative importance of the various physics involved in aircraft gust encounters and the effects of different modeling assumptions. The study demonstrates the importance of modeling rigid body motion, pitch dynamics, and aircraft flexibility, but shows that for gust encounters at typical flight conditions, a quasisteady aerodynamics assumption is reasonable for load predictions over the majority of the wing acerage, meaning that full unsteady aerodynamic simulations are likely not required for typical gust lengths.
\end{abstract}

\section{Introduction}

In order to meet air capacity goals without compromising environmental impact, future transport aircraft must satisfy increasingly stringent and diverse requirements that include constraints on fuel burn, emissions, and noise. Advanced technologies, such as new materials, constructive use of aeroelasticity, and active management of aircraft loads are already being incorporated into commercial aircraft; future environmental prospects demand a step change, which can only be achieved by more aggressive deployment of such technologies together with novel aircraft configurations. In this paper, we consider the specific problem of dynamic loading constraints due to maneuver and gust loads, which are an important design consideration for novel aircraft configurations that employ high aspect ratios, advanced structural layouts, and/or active management of aircraft loads.

Incorporating dynamic loading constraints in the early design process with an appropriate level of fidelity presents a significant challenge. The first challenge is the tight coupling between aerodynamics and control. In the conceptual design phase, which traditionally relies on low-fidelity empirical models, simple aerodynamic models may not capture the essential flow physics. Further, definitions of the control architecture, sensor placement, actuation models, etc. are typically not available early in the design process. A second challenge is the stochastic nature of the gust encounter problem, which must be carefully considered when optimal control strategies are used for load reduction or when optimizing the wing design. For example, simulation of several gust profiles will be needed at each optimization step, including an element of randomness to ensure that the optimizer does not anticipate and/or exploit knowledge of the gust shape. The controller design process must include not just the power spectral densities specified in the Federal Aviation Regulations, but also appropriate random amplitudes and phases in the combined spectrum. In addition, there are nonlinearities associated with section stall, surface deflection, and actuator rate limits that must be included.

\footnotetext{
*Assistant Professor, AIAA Member, Department of Aeronautics and Astronautics, University of Michigan.

$\dagger$ Technical Fellow, The Boeing Company, Seattle, Washington, Senior Member AIAA

¥Associate Professor, AIAA Associate Fellow, Department of Aeronautics and Astronautics, Massachusetts Institute of Technology.

$\S$ Professor, AIAA Fellow, Department of Aeronautics and Astronautics, Stanford University.
} 
The objective of this work is to propose an approach to multidisciplinary design optimization that incorporates dynamic load constraints, including the effects of stochastic gusts, as illustrated in Figure 1. The specific contribution of this paper is to define a gust modeling approach that is suitable for the optimization setting, with a particular focus on two challenges: first, to handle the stochastic element in an effective manner, and second, to address the need for computational efficiency. We address the first challenge by using Lyapunov and matched filter theory approaches, which allow explicit consideration of uncertainty and provide rigorous bounds for the linear case. We address the second challenge through a set of gust simulation studies that assess the relative importance of the various physics involved in aircraft gust encounters and the effects of different modeling assumptions.

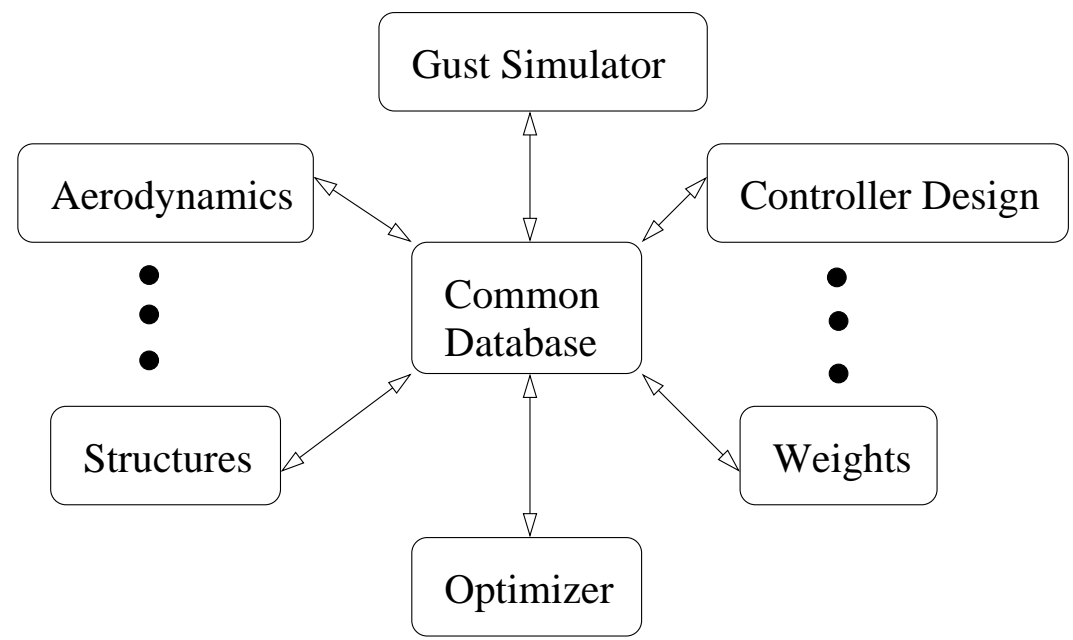

Figure 1. Optimization framework for aircraft design studies, ${ }^{1}$ showing modules related to active gust load alleviation.

Section II of the paper presents the three gust analysis approaches that are considered in this work: direct power spectral density integration, a Lyapunov equation approach, and matched filter theory. These methods are implemented and compared for a simple two degree of freedom aircraft model. In Section III, the gust analysis methods are applied to a realistic dynamical system obtained from a coupled aerodynamics and structural simulation code. Discrete gust encounter simulations with the same code additionally give insight into the physics of gust responses. Finally, we present conclusions and directions of ongoing work in Section IV.

\section{Gust Analysis Approaches}

\section{II.A. Background}

Gust-load alleviation has been a long-time research area in aircraft analysis and design. Nissim et al. combined analytical aerodynamic models with forward-in-time numerical simulations to analyze the effect of inboard control strips on rigid body motions caused by discrete gust encounters. ${ }^{2}$ Swaim et al. present a full-state gain feedback gust-load alleviation control strategy that uses a gust prefilter and requires solution of a Riccati equation to minimize the mean square of a load of interest. ${ }^{3}$ Nam et al. consider a full multidisciplinary design optimization of a wing, incorporating a gust-load alleviation system through sizable control surfaces in addition to shape and structural design variables such as planform dimensions and composite ply orientation. ${ }^{4}$ An alternate design strategy is presented by Aouf et al. , who consider robust control design for a B-52 aircraft model with flexible modes. ${ }^{5}$

Related research also exists on incorporating control surface deflection into design. Kolonay et al. consider the use of active control surface deflections to minimize drag at off-design cruise conditions. ${ }^{6}$ Thompson et al. present a novel analysis technique for induced drag minimization, in which trailing-edge surfaces are simulated via transpiration boundary conditions, thus eliminating the need for grid motion in an Euler finite volume solver. ${ }^{7}$

This work will focus on developing an analysis approach that takes into account the stochastic nature of 
atmospheric gusts and that is suitable for use in optimization. The following subsections setup the problem and review two gust options for gust analysis: one based on solving Lyapunov equations, and another based on matched filter theory. In addition, these approaches are validated for a simple two-degree of freedom aircraft in the last subsection.

\section{II.B. Problem setup}

The Federal Aviation Regulations (FAR) require transport aircraft to be analyzed by discrete gust and continuous turbulence analyses. ${ }^{8}$ The discrete gust is represented by a single 1-cos gust profile in the time domain with varying gust gradient length while the continuous turbulence is defined by the statistical measures of the Von Karman spectrum in the frequency domain. The continuous turbulence requirement is further divided into the design envelope and the mission analysis criteria.

In the design envelope analysis the root-mean-square (RMS) response due to unit RMS gust, $\bar{A}$, is first calculated and then scaled by a design gust velocity, $U_{\sigma}$ and added to the 1 -g mean response, $y_{1 g}$, giving

$$
y_{\text {design }}=\bar{A} U_{\sigma}+y_{1 g} .
$$

Here, a response refers to an output of interest such as bending moment, acceleration, deflection, etc. In the mission analysis, the RMS responses are first calculated for a number of flight segments in a typical flight mission. Exceedance curves are generated accounting for the duration of each flight segment $t_{i}$, the storm and non-storm gust intensities in each segment $\left(b_{1, i}\right.$ and $\left.b_{2, i}\right)$ and the probability of encountering those gust intensities $\left(P_{1, i}\right.$ and $\left.P_{2, i}\right)$. From the exceedance curves, a limit design load is defined as the value that is exceeded $2 \times 10^{-5}$ times per hour.

A key challenge addressed within this project is to incorporate the gust analysis in the design optimization setting. Specifically, we must address the problem of how to represent the stochastic nature of the problem. If we were to use a 1-cos gust profile, the optimization would anticipate the gust and choose optimal design variable values to exploit this knowledge. The resulting design would be optimized for the 1-cos gust, but otherwise practically useless when subjected to other gust excitations. Other methods to determine the gust limit design value (such as Monte Carlo sampling) are too expensive to be used within the optimization context. Our approach will be to use linear gust analysis methods within the optimization loop, and then check nonlinear effects a posteriori. A linear analysis is used to exploit the structure of the dynamical system and allows us to take advantage of efficient analysis methods.

In the following, two approaches are presented for analyzing linear airplane models subjected to gust excitation. The complete system consists of input white noise, $w_{w}(t)$, passing through a gust filter to produce a gust velocity, $w_{g}(t)$, as illustrated in Figure 2. The prefilter dynamics are given by

$$
\begin{aligned}
\left\{\dot{x}_{g}(t)\right\} & =\left[A_{g}\right]\left\{x_{g}(t)\right\}+\left\{B_{g}\right\} w_{w}(t) \\
w_{g}(t) & =\left[C_{g}\right]\left\{x_{g}(t)\right\}
\end{aligned}
$$

where $\left\{x_{g}\right\}$ is the state vector of gust variables and the matrices $\left[A_{g}\right],\left\{B_{g}\right\}$, and $\left[C_{g}\right]$ constitute a realization of the gust filter. The aircraft dynamics are given by

$$
\begin{aligned}
\{\dot{x}(t)\} & =[A]\{x(t)\}+\{B\} w_{g}(t), \\
y(t) & =[C]\{x(t)\},
\end{aligned}
$$

where $\{x\}$ is the aircraft state vector, $y$ is the output response, and the matrices $[A],\{B\}$, and $[C]$ define the aircraft dynamics. The complete input-to-output dynamics can be written as

$$
\begin{aligned}
\left\{\dot{x}_{w}(t)\right\} & =\left[A_{w}\right]\left\{x_{w}(t)\right\}+\left\{B_{w}\right\} w_{w}(t), \\
y(t) & =\left[C_{w}\right]\left\{x_{w}(t)\right\}
\end{aligned}
$$

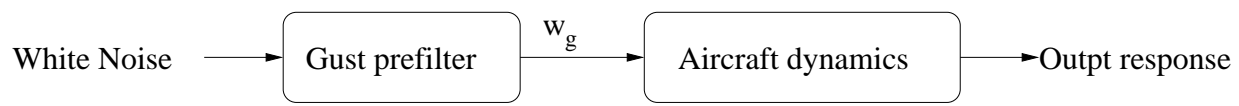

Figure 2. Schematic of the white noise to output mapping used for linear gust analysis. 
where

$$
\left\{x_{w}\right\}=\left\{\begin{array}{c}
x_{g} \\
x
\end{array}\right\}, \quad\left[A_{w}\right]=\left[\begin{array}{cc}
A_{g} & 0 \\
B C_{g} & A
\end{array}\right], \quad\left\{B_{w}\right\}=\left\{\begin{array}{c}
B_{g} \\
0
\end{array}\right\} .
$$

Alternatively, defining $G_{g w}(s)$ as the prefilter transfer function and $H_{y g}(s)$ as the aircraft transfer function, the input-to-output dynamics can be written as

$$
Y(s)=H_{y w}(s) W_{w}(s)=H_{y g}(s) G_{g w}(s) W_{w}(s),
$$

where $s$ is the Laplace variable and $Y(s)$ denotes the Laplace transform of $y(t)$.

\section{II.C. Lyapunov Equation Approach}

For a linear, stable aircraft model excited by white noise, the design value of the state covariance matrix $\left[\Gamma_{w}\right]$ can be calculated directly by solving the Lyapunov equation, ${ }^{9-11}$

$$
\left[A_{w}\right]\left[\Gamma_{w}\right]+\left[\Gamma_{w}\right]\left[A_{w}\right]^{T}=-\left\{B_{w}\right\} U_{\sigma}^{2}\left\{B_{w}\right\}^{T},
$$

where $U_{\sigma}$ is the gust velocity. The design gust response then becomes

$$
y_{\text {design }}=\sqrt{\left[C_{w}\right]\left[\Gamma_{w}\right]\left[C_{w}\right]^{T}}+y_{1 g}
$$

The advantage of this approach is that the covariance matrix and the root-mean-square response can be calculated without numerical integration in the frequency domain. Livne showed that the Lyapunov equation can be solved efficiently with an alternate approach based on the eigenvectors and eigenvalues of the system. ${ }^{11}$ The complex right eigenvectors and eigenvalues of the $\left[A_{w}\right]$ matrix are calculated and the eigenvectors are first assembled in a matrix,

$$
\left\{\psi_{i}\right\} \lambda_{i}=\left[A_{w}\right]\left\{\psi_{i}\right\} \quad[\Psi]=\left[\psi_{1}, \psi_{2}, \ldots, \psi_{N}\right],
$$

where $\left\{\psi_{i}\right\}$ is the $i^{\text {th }}$ eigenvector of $\left[A_{w}\right]$ and $\lambda_{i}$ is the corresponding eigenvalue. Note that the set of eigenvectors includes aeroservoelactic modes since the full dynamics of the aircraft is modeled. It can then be shown that the state covariance matrix can be calculated from

$$
\left[\Gamma_{w}\right]=[\Psi][D][\Psi]^{T},
$$

where the $i, j$ term of the matrix $[D]$ is calculated from

$$
D_{i j}=\frac{\left(-[\Psi]^{-1}\left\{B_{w}\right\} U_{\sigma}^{2}\left\{B_{w}\right\}^{T}[\Psi]^{-T}\right)_{i j}}{\lambda_{i}+\lambda_{j}} .
$$

Due to the presence of aeroservoelastic eigenvalues in the denominator of (2), the state covariance matrix becomes infinite when the damping of any of the aeroelastic modes approaches zero. This phenomenon was observed and utilized in generating efficient approximations for the state covariance for use in gradient-based design optimization. ${ }^{11,12}$

Another advantage of the Lyapunov equation approach is that the analytical sensitivities of the state covariance matrix (and hence the design gust response) to a design variable $p_{j}$ can be found by solving another Lyapunov equation:

$$
\left[A_{w}\right]\left[\frac{d \Gamma_{w}}{d p_{j}}\right]+\left[\frac{d \Gamma_{w}}{d p_{j}}\right]\left[A_{w}\right]^{T}=-\left\{\frac{d B_{w}}{d p_{j}}\right\} U_{\sigma}^{2}\{\tilde{B}\}^{T}-\{\tilde{B}\} U_{\sigma}^{2}\left\{\frac{d B_{w}}{d p_{j}}\right\}^{T}-\left[\frac{d A_{w}}{d p_{j}}\right]\left[\Gamma_{w}\right]-\left[\Gamma_{w}\right]\left[\frac{d A_{w}}{d p_{j}}\right]^{T} .
$$

\section{II.D. Matched Filter Theory}

In the matched filter theory (MFT) approach, ${ }^{13-17}$ the white noise excitation input is replaced by a waveform $w_{x}(t)$ that will result in a worst case gust response:

$$
Y(i \omega)=H_{y w}(i \omega) W_{x}(i \omega)
$$


where $Y(i \omega)$ is the Fourier-transform of the output, $W_{x}(i \omega)$ is the Fourier-transform of $w_{x}(t)$, and $H_{y w}$ is the white noise to output transfer function. The waveform that produces the worst-case gust response can be found using the following approach and is called the matched excitation waveform.

The matched excitation waveform is calculated as the normalized unit impulse response of the system $H_{y w}(s)$ reversed in time and shifted by time $t_{0},{ }^{13}$

$$
w_{x}(t)=h_{y}\left(t_{0}-t\right) / K
$$

where the unit impulse response is:

$$
h_{y}(t)=\frac{1}{2 \pi} \int_{-\infty}^{\infty} H_{y w}(i \omega) e^{i \omega t} d \omega
$$

The constant $K$ is chosen to be equal to the standard deviation of the impulse response,

$$
K=\sigma_{h_{y}}=\sqrt{\frac{1}{2 \pi} \int_{-\infty}^{\infty} H_{y w}(-i \omega) H_{y w}(i \omega) d \omega}
$$

such that the energy of the matched filter is unity.

The MFT approach has been proposed as a method for calculating gust loads since the late 80's in a paper by Zeiler and Pototzky. ${ }^{13}$ The promise of the MFT is that it would not only generate a gust response time history with a worst-case peak, but also the gust excitation waveform that produced this peak. In the 1990s there was a focus on showing the relationship between the MFT and the Power Spectral Density (PSD) approach and exploring extension of MFT to include nonlinear effects. ${ }^{14,17}$

From the above definitions

$$
W_{x}(\omega)=\int_{-\infty}^{\infty} w_{x}(t) e^{-i \omega t} d t=\frac{1}{\sigma_{h_{y}}} \int_{-\infty}^{\infty} h_{y}\left(t_{0}-t\right) e^{-i \omega t} d t=\frac{1}{\sigma_{h_{y}}} H_{y w}(-i \omega) e^{-i \omega t_{0}} .
$$

The gust response time history is defined by

$$
y(t)=\frac{1}{2 \pi} \int_{-\infty}^{\infty} Y(i \omega) e^{i \omega t} d \omega=\frac{1}{2 \pi} \int_{-\infty}^{\infty} H_{y w}(i \omega) W_{x}(i \omega) e^{i \omega t} d \omega=\frac{1}{\sigma_{h_{y}}} R_{y y}\left(t-t_{0}\right),
$$

where $R_{y y}(\tau)$ is the autocorrelation function

$$
R_{y y}(\tau)=\frac{1}{2 \pi} \int_{-\infty}^{\infty} S_{y y}(\omega) e^{i \omega \tau} d \omega
$$

and $S_{y y}=H_{y w}(i \omega) H_{y w}(-i \omega)$ is the response spectrum of $y(t)$ due to white noise excitation.

Eqn. 3 states that the output gust response time history is proportional to the autocorrelation function centered on time $t_{0}$. The autocorrelation function is an even function centered at $\tau=0$, with a maximum value at $\tau=0$ equal to the variance of the impulse response, $R_{y y}(0)=\sigma_{h_{y}}^{2}$, and diminishing to zero for extreme positive and negative values of $\tau$. The response time history then yields a maximum of $\sigma_{h_{y}}$ at time equal to $t_{0}$

$$
y_{\max }=y\left(t=t_{0}\right)=\sigma_{h_{y}} .
$$

The maximum of the PSD gust response is also defined as the RMS value $\sigma_{h_{y}}$ and is found as the square-root of the integrated value of the response spectrum over all frequencies. In other words, the MFT response time history is the autocorrelation function offset with a time value of $t_{0}$.

The gust excitation wave form that generates $y_{\max }=y\left(t=t_{0}\right)=\sigma_{h_{y}}$ can be calculated from

$$
w_{g y}(t)=\frac{1}{2 \pi} \int_{-\infty}^{\infty} G_{g w}(i \omega) W_{x}(i \omega) e^{i \omega t} d \omega=\frac{1}{\sigma_{h_{y}}} \frac{1}{2 \pi} \int_{-\infty}^{\infty} G_{g w}(i \omega) H_{y w}(-i \omega) e^{i \omega\left(t-t_{0}\right)} d \omega=\frac{1}{\sigma_{h_{y}}} R_{g y}\left(t-t_{0}\right),
$$

where $R_{g y}()$ is the cross-correlation function between the gust velocity $w_{g}(t)$ and the response $y(t)$. The gust excitation $w_{g y}(t)$ will only ensure that the response $y(t)$ reaches a maximum of $\sigma_{h_{y}}$ at $t=t_{0}$. Another output quantity, $z(t)$ will reach a maximum value with a different gust excitation waveform. In other words, each response quantity will be maximized by a unique gust excitation waveform.

An output response $z(t)$ that is time correlated with $y(t)$ is calculated from

$$
z_{y}(t)=\frac{1}{\sigma_{h_{y}}} \frac{1}{2 \pi} \int_{-\infty}^{\infty} H_{z w}(i \omega) H_{y w}(-i \omega) e^{i \omega\left(t-t_{0}\right)} d \omega=\frac{1}{\sigma_{h_{y}}} R_{z y}\left(t-t_{0}\right),
$$

i.e. proportional to the cross-correlation function between $z(t)$ and $y(t)$. 


\section{II.E. Analysis Method Comparison}

To test the gust analysis approaches presented in the previous section, a simple two-degree of freedom aircraft model was created. The model is illustrated in Figure 3 and the nominal specifications are given in Table 1. The equations of motion for this model are as follows:

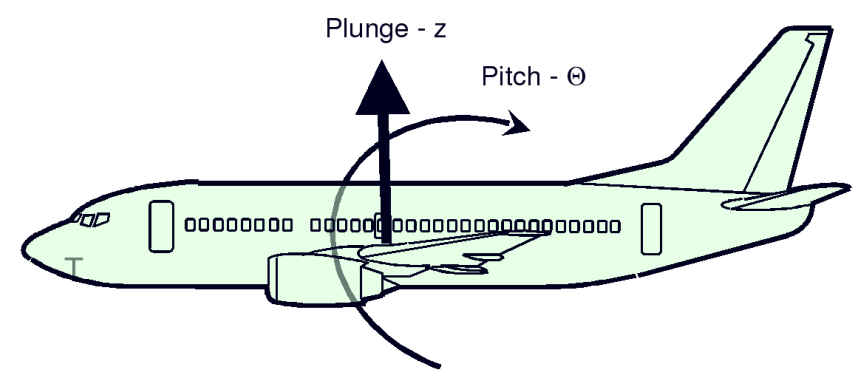

Figure 3. A simplified aircraft model with two degrees of freedom: pitch $(\theta)$ and plunge $(z)$.

Table 1. Nominal aircraft specifications for the two degree of freedom model.

\begin{tabular}{|cc|c|}
\hline$W$ & $:$ aircraft weight & $100,000 \mathrm{lb}$ \\
$r_{y}$ & $:$ pitch inertia radius of gyration & $400 \mathrm{in}$ \\
$S$ & $:$ wing reference area & $1000 \mathrm{ft}^{2}$ \\
$\bar{c}$ & $:$ reference chord & $150 \mathrm{in}$ \\
$V$ & $:$ aircraft forward speed & $800 \mathrm{ft} / \mathrm{s}$ \\
$C_{l \alpha}$ & $:$ lift curve slope & $7.0 \mathrm{rad}^{-1}$ \\
$C_{m \alpha}$ & $:$ pitching moment slope & $-3.0 \mathrm{rad}^{-1}$ \\
$C_{l q}$ & $:$ lift curve due to pitch rate & $10 \bar{c} /(2 \mathrm{~V}) 1 /(\mathrm{rad} / s)$ \\
$C_{m q}$ & $:$ pitching moment due to pitch rate & $\bar{c} /(2 \mathrm{~V}) 1 /(\mathrm{rad} / s)$ \\
$r_{1}$ & $:$ root bending moment coefficient (scales lift) & $100 \mathrm{in}$ \\
$r_{2}$ & $:$ root bending moment coefficient (scales mass) & $20 \mathrm{in}$ \\
$r_{3}$ & $:$ distance between pilot station and center of gravity & $400 \mathrm{in}$ \\
\hline
\end{tabular}

$$
\underbrace{\frac{W}{q_{\infty} S g}\left[\begin{array}{cc}
1 & 0 \\
0 & r_{y}^{2} / \bar{c}
\end{array}\right]}_{\mathbf{M}}\left[\begin{array}{c}
\ddot{z} \\
\ddot{\theta}
\end{array}\right]=\underbrace{\left[\begin{array}{cc}
0 & C_{l \alpha} \\
0 & C_{m \alpha}
\end{array}\right]}_{\mathbf{A}_{0}}\left[\begin{array}{c}
z \\
\theta
\end{array}\right]+\underbrace{\left[\begin{array}{cc}
-C_{l \alpha} / V & C_{l q} \\
-C_{m \alpha} / V & C_{m q}
\end{array}\right]}_{\mathbf{A}_{1}}\left[\begin{array}{c}
\dot{z} \\
\dot{\theta}
\end{array}\right]+\underbrace{\left[\begin{array}{c}
C_{l \alpha} / V \\
C_{m \alpha} / V
\end{array}\right]}_{\mathbf{A}_{g}}\left[\alpha_{g}\right]
$$

where $g$ is the acceleration due to gravity, $q_{\infty}$ is the dynamic pressure, $\alpha_{g}$ is the gust-induced perturbation in the angle of attack, and the other notation is defined in Table 1. This equation can be reduced to state-space form by defining

$$
\mathbf{x}_{1}=\left[\begin{array}{c}
z \\
\theta
\end{array}\right], \quad \mathbf{x}_{2}=\dot{\mathbf{x}}_{1}
$$

resulting in,

$$
\left[\begin{array}{c}
\dot{\mathbf{x}}_{1} \\
\dot{\mathbf{x}}_{2}
\end{array}\right]=\left[\begin{array}{cc}
0 & \mathbf{I} \\
\mathbf{M}^{-1} \mathbf{A}_{0} & \mathbf{M}^{-1} \mathbf{A}_{1}
\end{array}\right]\left[\begin{array}{l}
\mathbf{x}_{1} \\
\mathbf{x}_{2}
\end{array}\right]+\left[\begin{array}{c}
0 \\
\mathbf{M}^{-1} \mathbf{A}_{g}
\end{array}\right]\left[\begin{array}{l}
\alpha_{g}
\end{array}\right]
$$

The two outputs of interest in this sample problem are the root bending moment and the pilot acceleration. These are lumped into one vector $\mathbf{y}$, as follows:

$$
\mathbf{y}=\left[\begin{array}{c}
\text { Root bending moment } \\
\text { Pilot acceleration }
\end{array}\right]=\left[\begin{array}{c}
q_{\infty} S r_{1} C_{l \alpha}\left(\theta-\frac{\dot{z}}{V}+\alpha_{g}\right)-r_{2} \frac{W}{g} \ddot{z} \\
\ddot{z}+r_{3} \ddot{\theta}
\end{array}\right] .
$$


This expression can be written in terms of $\mathbf{x}_{1}, \mathbf{x}_{2}$, and $\alpha_{g}$, by using Eqns. 4 and 5 .

With the above state space system, stochastic gust analyses were performed using the methods described in Section II. For success of the Lyapunov approach, the model was modified by grounding the aircraft using soft springs. This addition was necessary to stabilize modes with roots on the imaginary axis. Critical damping was also added to these modes to make them well-behaved. For the Von Karman spectrum/gust filter, a gust length of $L=2500 \mathrm{ft}$ and a gust velocity of $U_{\sigma}=75 \mathrm{ft} / \mathrm{s}$ were used.

A comparison of the RMS outputs obtained using the various approaches is shown in Table 2. The results are very similar for the different methods. Differences can be attributed to the number of frequency samples used in the numerical frequency integration and to the number of samples used in the discrete Fourier transforms for the matched filter theory approach.

Table 2. RMS outputs for the two degree of freedom aircraft.

\begin{tabular}{|c|c|c|}
\hline Method & Bending moment $\left(10^{6}\right.$ in-lb) & Pilot acceleration $\left(\mathrm{in} / \mathrm{s}^{2}\right)$ \\
\hline Frequency integration (spectrum) & 20.256 & 824.33 \\
Frequency integration (filter) & 20.127 & 819.16 \\
Lyapunov equation & 20.291 & 825.78 \\
Matched filter theory & 20.614 & 828.76 \\
\hline
\end{tabular}

Out of the methods tested, the direct frequency integration approach is the most expensive, as the numerical integration requires sampling the output response at a large number of frequencies. Solving the Lyapunov equations or calculating a single system impulse response for the MFT approach are much less expensive, and this effect is especially important for large systems. In addition, the Lyapunov approach has the advantage of an analytical equation for sensitivity calculations, while the MFT approach yields a worst-case gust profile that can be incorporated into optimization.

\section{Application to a Coupled Aerodynamics Structural Model}

The stochastic gust analysis methods were applied to a large system obtained from a coupled aerodynamics and structural simulation of a realistic aircraft. The following sections describe the aircraft model and the associated stochastic gust analysis.

\section{III.A. Problem Setup}

A coupled aerodynamics-structural simulation of a transport aircraft was performed in ASWING, which is a configuration development code for flexible aircraft. ${ }^{18}$ ASWING features a finite difference discretization of the Euler-Bernoulli beam equations for each structure (fuselage, wing, tail) coupled with enhanced unsteady lifting line aerodynamics. The software is capable of time-accurate simulations via a second order backward difference discretization, and it allows for user-defined gust fields. For the simulations in this study, a Prandtl-Glauert compressibility correction is employed.

The aircraft model used for this study is based roughly on a Boeing 737-400 transport aircraft. The model geometry, along with the finite difference discretization sections, is illustrated in Figure 4. The weight distribution was chosen approximately to yield an adequate static stability margin, including point masses for the landing gear and engines. The wing stiffness distribution varied exponentially from root to tip and was calibrated using wing tip deflection at cruise.

The coupled aerodynamics-structural finite-difference discretization results in an unsteady residual equation at each time step of the form

$$
\mathbf{r}(\mathbf{x}, \dot{\mathbf{x}})=0
$$

where $\mathbf{x}$ is the state vector composed of the structural state variables of each beam section, the aerodynamic circulation coefficients for each lifting surface, and global variables such as beam joint moments, aircraft position and velocity, etc. For the simulations in this work, a total of 1261 degrees of freedom were used. Note, not all components of $\mathbf{x}$ appear as rates in the residual equations. 


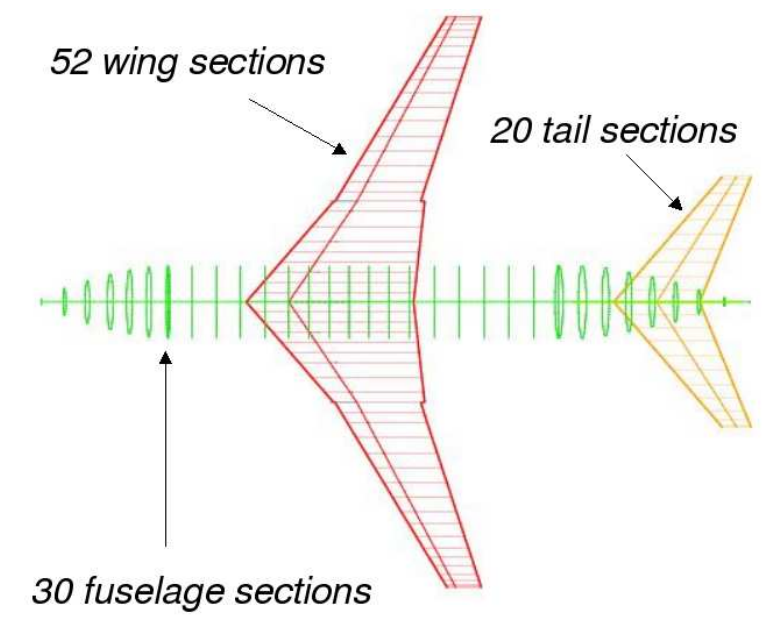

Figure 4. ASWING model planform. Based on a Boeing 737-400.

\section{III.B. Gust Encounter Simulations}

One of the contributions of this paper is to document a set of detailed simulation studies that assess the relative importance of the various physics involved in aircraft gust encounters. This assessment then leads us to a simplified analysis approach that retains the key physics at the required fidelity (to ensure realistic designs), but gives a model that is computationally tractable and suitable for use within a design optimization setting.

Therefore, prior to stochastic gust analyses, a detailed set of discrete gust-encounter simulations were performed using vertical " 1 -cos" gusts, in which the gust profile is given by

$$
V_{z}=\frac{V_{z, \max }}{2}\left(1-\cos \left(\frac{\pi d}{H}\right)\right), \quad \text { for } 0 \leq d \leq 2 H,
$$

where $V_{z, \max }$ is the maximum gust velocity, $H$ is the gust gradient length, and $d$ is the distance into the gust. Contours of a sample gust profile are shown in Figure 5. For the initial condition, the aircraft was trimmed for level flight at 20,000 ft altitude and $800 \mathrm{ft} / \mathrm{s}$ true air speed. A constant $V_{z, \max }=40 \mathrm{ft} / \mathrm{s}$ was used for all of the runs.

The gust encounter studies were used to determine the appropriate level of modeling required (e.g. unsteady versus quasi-steady analysis), for determining the range of gust lengths that are likely to be critical in the design, and for assessing the impact of using a flexible versus rigid wing model. The following sections summarize relevant results of these studies.

\section{Flexible Versus Rigid Wing}

A nearly-rigid wing was modeled by increasing the wing bending and torsional stiffnesses by a factor of 1000. A simulation of the rigid wing was performed for gust gradient lengths of $H=100,300,600,1000 \mathrm{ft}$. Figure 6 compares the wing root bending moment for the rigid and flexible cases. As shown, flexibility of the wing alleviates the root bending moment. The rigid wing exhibits a higher initial moment and a lack of oscillations following the passage of the gust.

\section{Free Versus Anchored Analysis}

An anchored analysis was performed by grounding the fuselage near the CG, preventing translation and rotation. Variation in elevation angle and vertical position was therefore zero. The wing remained flexible, however. Figure 7 compares a simulation for $H=100,300,600,1000 \mathrm{ft}$ to a free (unanchored) case. The motion of the aircraft in the dynamic simulation reduces the peak loads. 


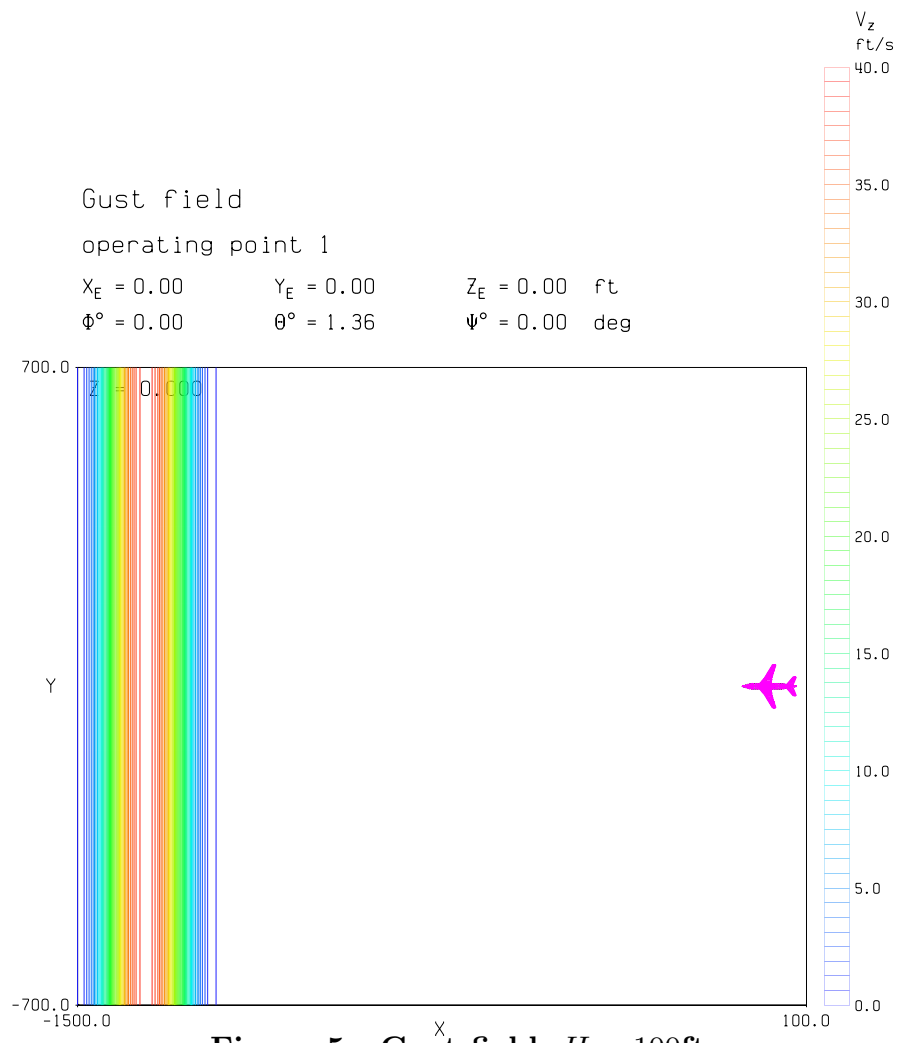

Figure 5. Gust field, $H=100 \mathrm{ft}$.

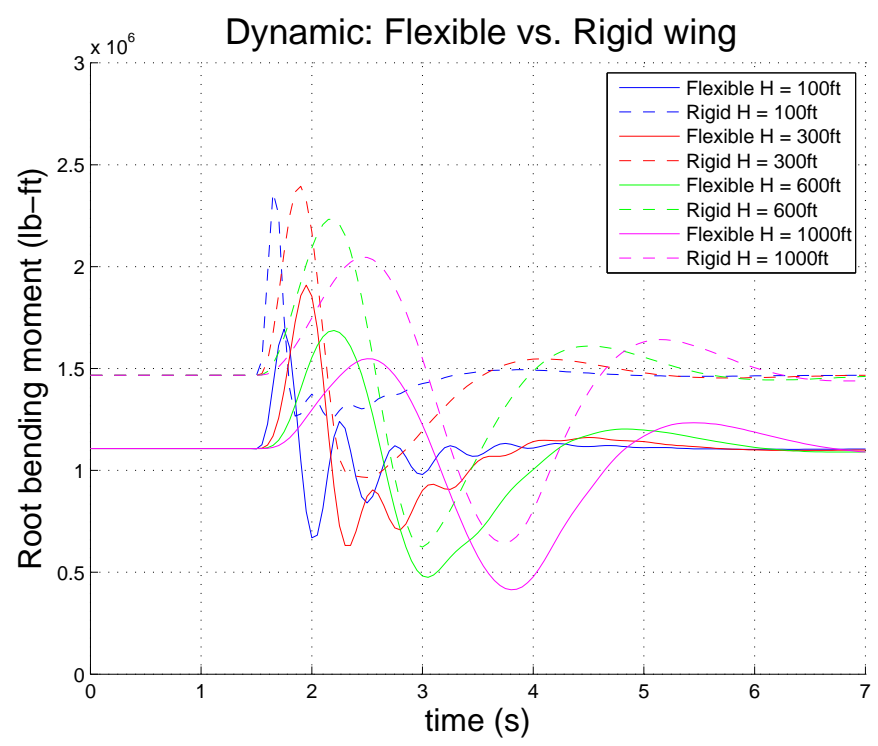

Figure 6. Dynamic simulation comparing a flexible wing versus a rigid wing. 


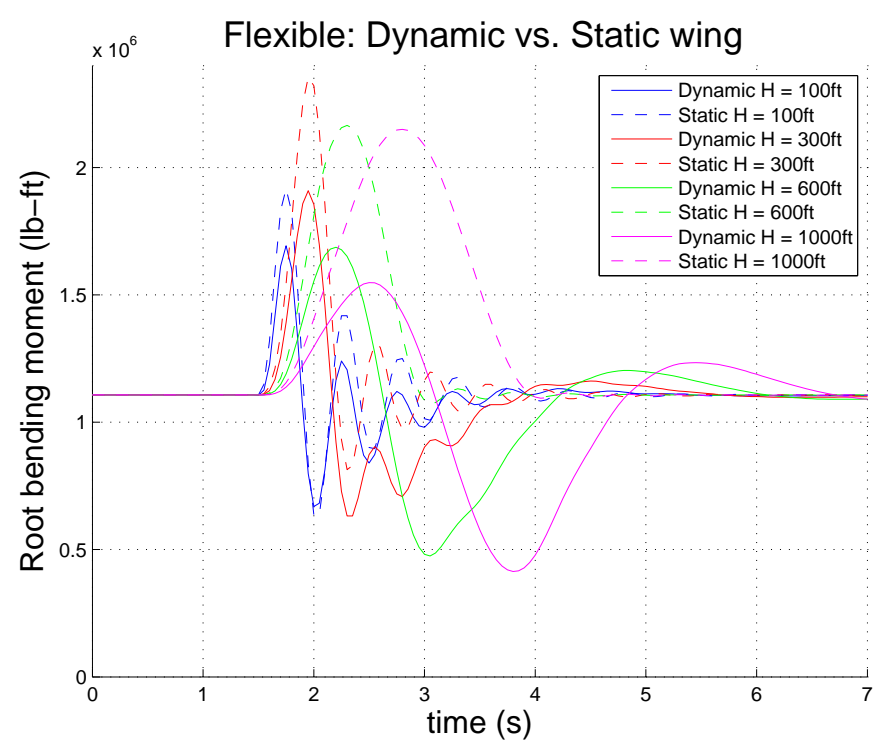

Figure 7. Flexible wing: dynamic simulation versus an anchored simulation.

Free Versus Plunge-Only Analysis

A plunge-only analysis was performed similarly to the anchored case, except that vertical displacement (plunge) was allowed. Variation in elevation angle was constrained to be zero. The wing remained flexible. Figure 8 compares a simulation for $H=100,300,600,1000 \mathrm{ft}$ to an free case. The agreement in the initial loads is good, and the extrema are captured. The smaller secondary oscillations are not captured accurately, especially for large $H$.

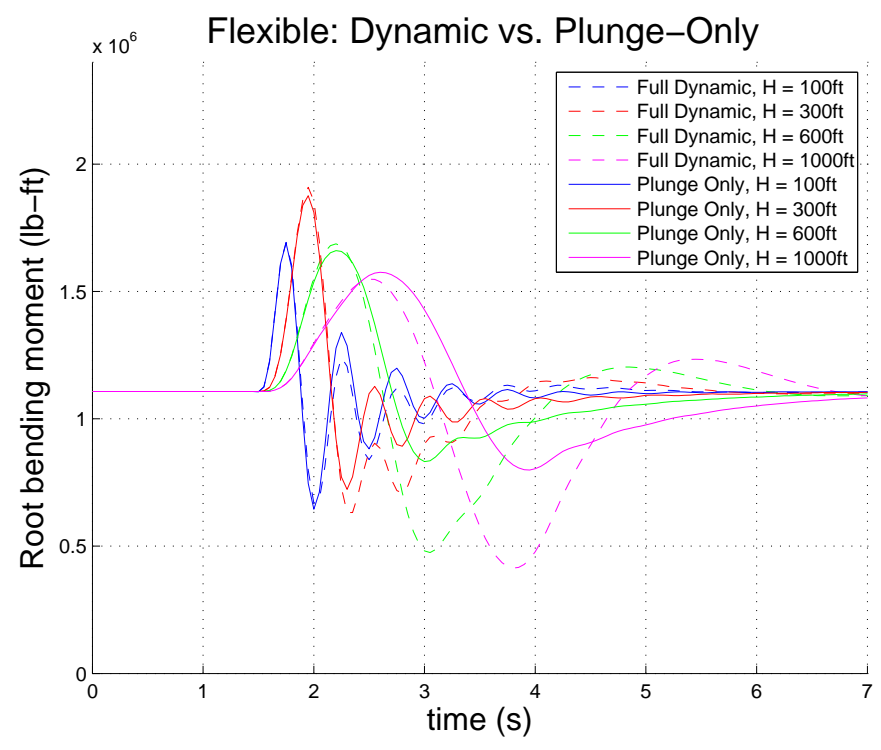

Figure 8. Flexible wing: dynamic simulation versus a plunge-only simulation.

\section{Effect of Unsteady Aerodynamics}

In ASWING, unsteady aerodynamic loads are computed using the unsteady vector form of the KuttaJoukowsky theorem, which takes into account the time rate of change of the bound vortices, $d \Gamma / d t$. However, assumptions are made when calculating the induced velocities. First, a flat, freestream-aligned wake is used, 
effectively assuming that the velocity variations of the aircraft are small compared to the freestream velocity. Second, unsteady shed vortices are assumed to only affect the surface that shed them. For example, the unsteady vortices shed from the main wing do not affect the induced velocity on the tail. However, the tail is still affected by the steady vortex sheet. Third, the unsteady downwash produced by a shed vortex from a surface is only modeled approximately. That is, integration is not performed over all previously shed vortices to compute the downwash. Rather, the effect of the shed vortices is modeled using an empirical lag term: a factor multiplying $d \Gamma / d t$ is added to the downwash on the surface. The factor is chosen to asymptotically match Theodorsen's $2 \mathrm{D}$ results for large reduced frequencies.

This study compares the results obtained with the unsteady lag term present ("unsteady aerodynamics") to those obtained with the lag term disabled ("quasi-steady aerodynamics"). As stated in the ASWING documentation, disabling the lag term contribution allows one to determine the importance of unsteady aerodynamics in a given application.

For the flexible aircraft, dynamic simulations were run with varying $H$, with unsteady aerodynamics turned off. Figure 9 compares the results to those using unsteady aerodynamics. The differences are small for the $H$ values considered.

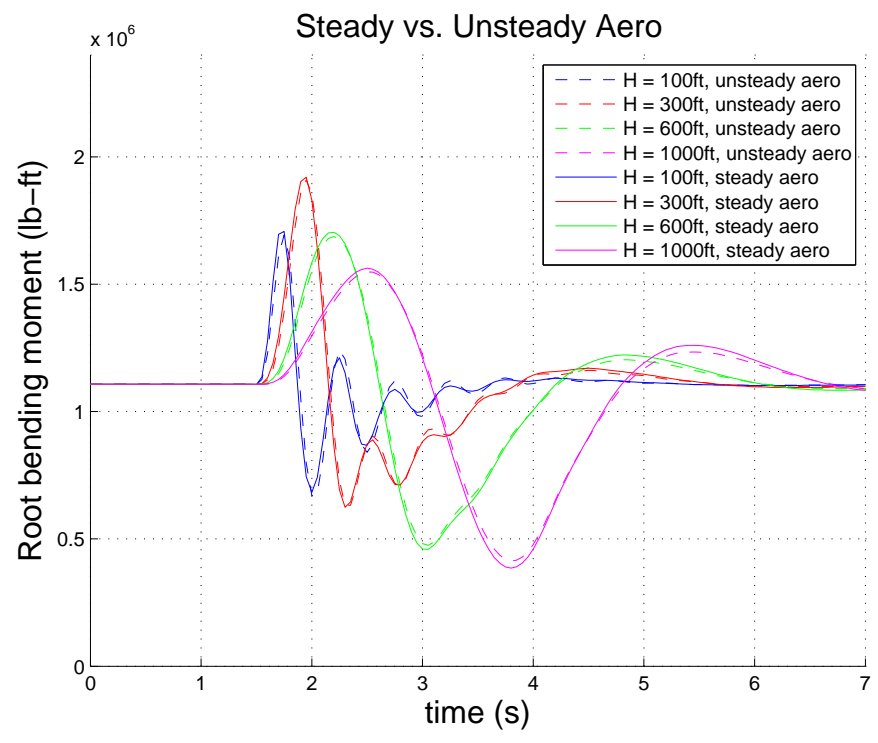

Figure 9. Quasi-steady versus unsteady aerodynamics.

\section{Short-Gust Simulations}

The previous sub-sections considered gust lengths $H \geq 100 \mathrm{ft}$. A short-gust study, with $H=30,50,100 \mathrm{ft}$, was also performed to assess the validity of the quasi-steady assumption and the criticalness of short gusts on outer portions of the wing. To study effects on the outer wing, the bending moment was measured not just at the root, but also at $2 y / b=0.5,0.75,0.9$, where $y$ is the spanwise coordinate, zero at the root, and $b$ is the full span.

Figure 10 shows the bending moment time histories at four locations along the wing, for the three gust lengths. The largest bending moment is observed for the $H=100 \mathrm{ft}$ gust for all locations. Quasi-steady aerodynamics results are also shown for each gust encounter simulation. Differences between the unsteady and quasi-steady results are present, but they are not large.

The results in Figure 10 show that the moment amplitude increases with $H$ for the three gust lengths considered. The response oscillation frequency, however, is approximately the same for all of the gust lengths, with the longer gust lengths lagging in phase behind the shorter gust lengths. A possible explanation for this effect is that all three gust lengths excite the same primary vibration mode(s) in the wing, with the excitement amplitude depending on the time spent in the gust. Specifically, as the aircraft enters the gust, the wing sections experience an increasing lift force due to the 1-cos gust profile. For the longer gust lengths, this forcing time is longer, leading to a greater impulse and a larger, delayed maximum moment before the free vibrations of the wing begin. 


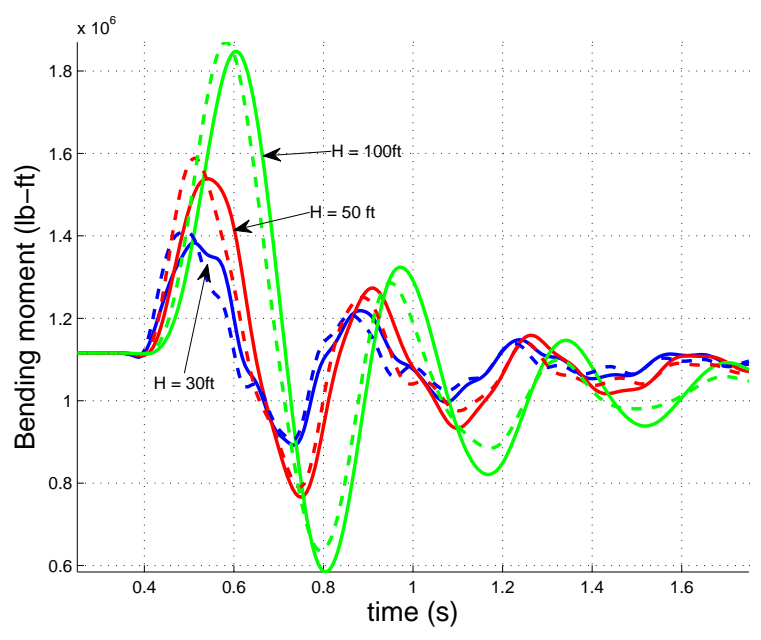

(a) $2 y / b=0$

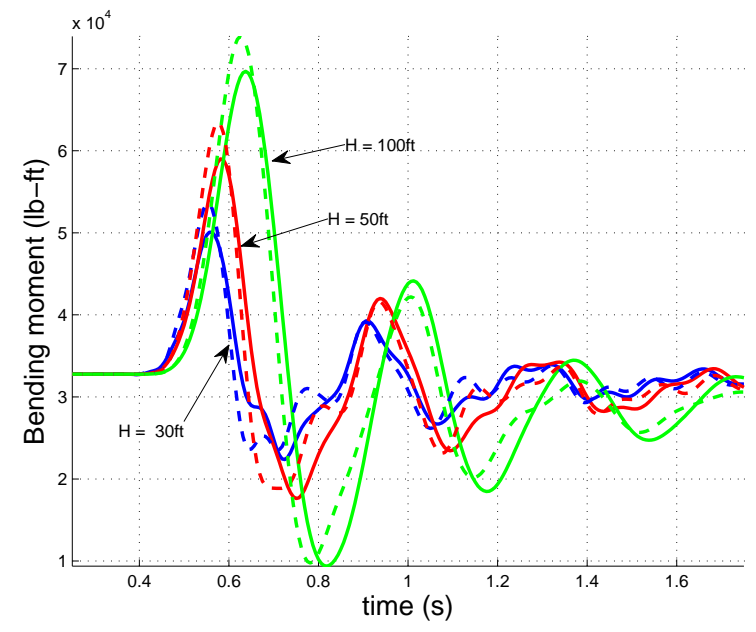

(c) $2 y / b=0.75$

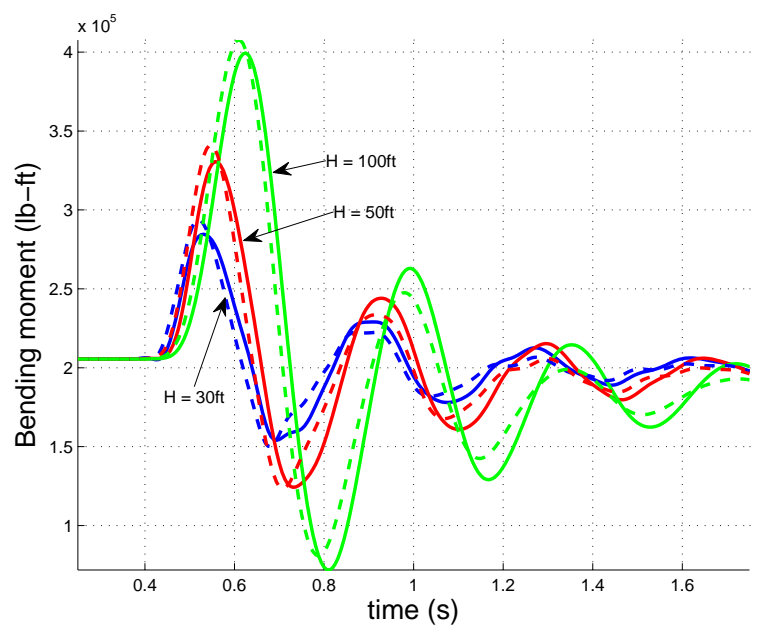

(b) $2 y / b=0.5$

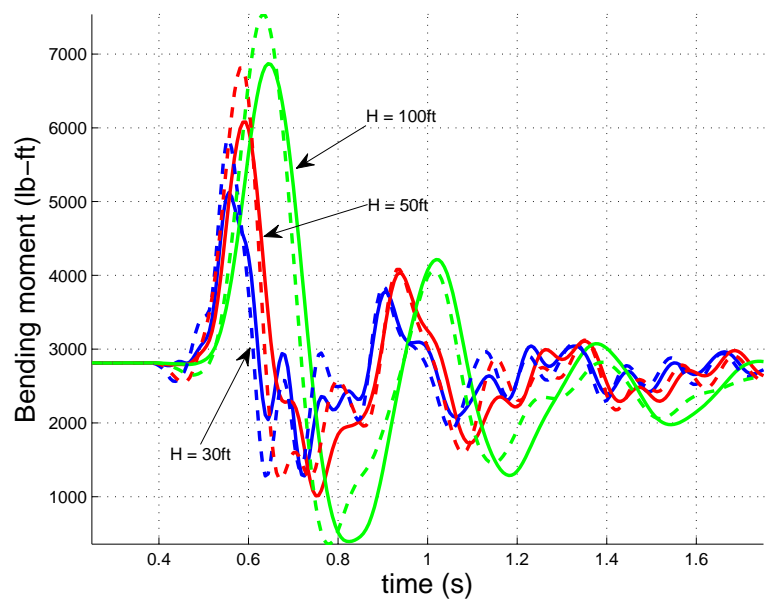

(d) $2 y / b=0.9$

Figure 10. Bending moment time histories at four span locations for $H=30,50,100 \mathrm{ft}$ gusts. Dashed lines represent quasi-steady results. Note the different ranges for the bending moment on the plots. 


\section{Gust Encounter Study Summary}

A 1-cos gust encounter for an approximate Boeing 737-400 model was simulated using ASWING. These simulations provide insight into the variation of the wing response with respect to gust length, the importance of pitch dynamics, and the importance of unsteady aerodynamics. Key points from the analysis are:

- An anchored (static) analysis yields greatly-overestimated loads compared to a full dynamic analysis, which includes rigid body motion that relieves the gust loads. Hence, a dynamic analysis is crucial for accurate load prediction.

- Longitudinal dynamics becomes important at longer gust lengths, making a plunge-only assumption invalid. Thus, pitch dynamics should be included in simulations at long gust lengths.

- For gust encounters at typical flight conditions, a quasi-steady aerodynamics assumption appears reasonable for the majority of the wing acerage, at gust lengths $H>30 \mathrm{ft}$. Thus, full unsteady simulations are likely not required for typical gust lengths.

These conclusions provide a roadmap for the approximations that can be made when the gust analysis is incorporated into a design optimization. Of course, the gust simulations were performed on one "typical" transport aircraft. For significantly different designs the simulations should be repeated with a wide range of gust lengths to verify that the modeling assumptions still hold.

\section{III.C. Stochastic Gust Analysis Results}

For the stochastic gust analysis, the nonlinear equations in Eqn. 6 must first be linearized. This linearization is performed about a steady-state deformed state, $\mathbf{x}_{0}$; that is, we define $\mathbf{x}(t)=\mathbf{x}_{0}+\delta \mathbf{x}(t)$. The resulting equations are:

$$
\begin{aligned}
{[M] \delta \ddot{\mathbf{x}} } & =[A] \delta \mathbf{x}+\{B\} \alpha_{g} \\
\delta \mathbf{y} & =[C] \delta \mathbf{x}
\end{aligned}
$$

where $[M]=-\partial \mathbf{r} / \partial \dot{\mathbf{x}},[A]=\partial \mathbf{r} / \partial \mathbf{x}$, and $\{B\}$ is obtained from the derivative of $\mathbf{r}$ with respect to the aircraft vertical velocity. $\mathbf{y}$ consists of the bending moment at the root and $50 \%$ span location along the wing, and $[C]$ is obtained from the sensor feature in ASWING. Since not all components of $\mathbf{x}$ appear as rates in the residual equations, the mass matrix $[M]$ is singular, and hence the dynamics are represented by a descriptor system. Each matrix is stored in a sparse storage format.

Using the linearized system in Eqn. 7, two stochastic gust analyses were performed to calculate the RMS root bending moment. First, a direct integration of the output power spectral density was carried out, as described in Section II, yielding an RMS root bending moment of $\sigma_{y}=7.72 \times 10^{5} \mathrm{lb}-\mathrm{ft}$. While simple, such a direct integration is expensive for the large linear system under consideration.

A second stochastic gust analysis was performed using matched filter theory. In this approach, a normalized, reversed, and time-shifted system impulse response serves as the critical excitation waveform. The system impulse response was obtained by first calculating the impulse response of the gust filter, and then running a forward time simulation of the plant (i.e. the aircraft dynamics). Both the impulse response and the critical excitation waveform are shown in Figure 11.

Note that the critical excitation waveform is the input to the gust filter. The associated critical gust profile is then the corresponding output of the gust filter. The output response is the output of the aircraft dynamics plant to the critical gust profile. Both the critical gust profile and the root bending moment output response are shown in Figure 12. The shape of the critical gust profile, a downdraft followed suddenly by a sharp updraft, agrees with intuition. The maximum output value is $\sigma_{y}=7.74 \times 10^{5} \mathrm{lb}-\mathrm{ft}$, which is also the RMS output response according to matched filter theory. This value agrees well with the direct PSD integration.

According to matched-filter theory, the critical gust profile yields the maximum output response for all waveforms with the same energy in excitation space. To test the validity of the results, 1-cos gust profiles were created with various gust gradient lengths, $H$, and with amplitudes scaled so that each profile had the same energy as the critical gust profile in excitation space. The gust profiles along with the corresponding output responses are shown in Figure 13. The critical gust profile yields the maximum response, although this maximum value is not overly conservative. 


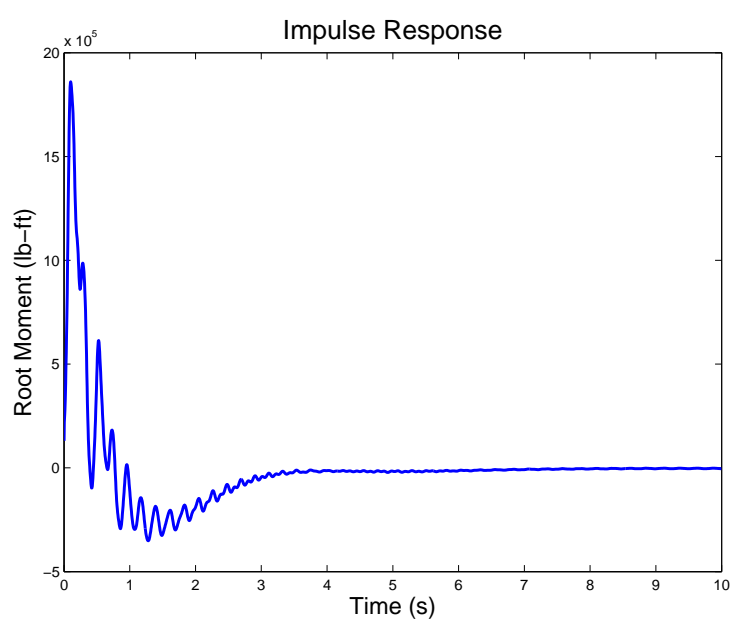

(a) Impulse response

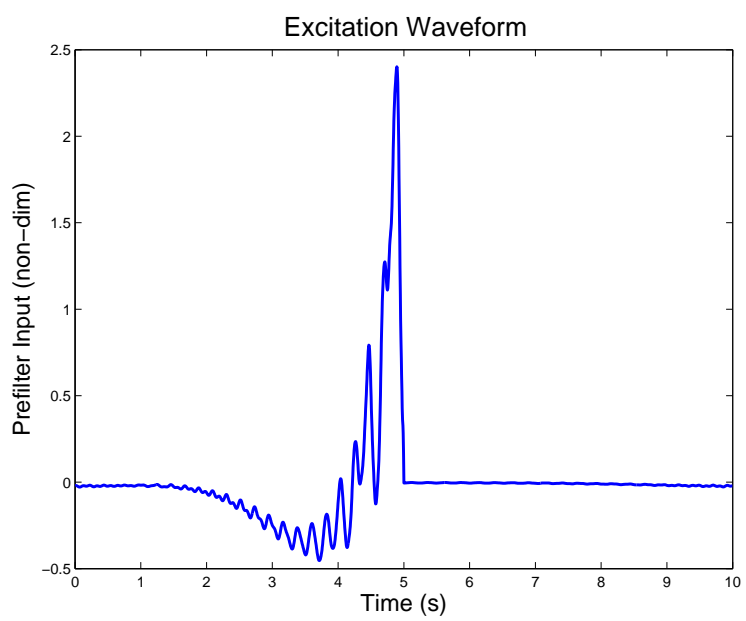

(b) Critical excitation waveform

Figure 11. Impulse response of ASWING model root bending moment, and the corresponding critical excitation waveform.

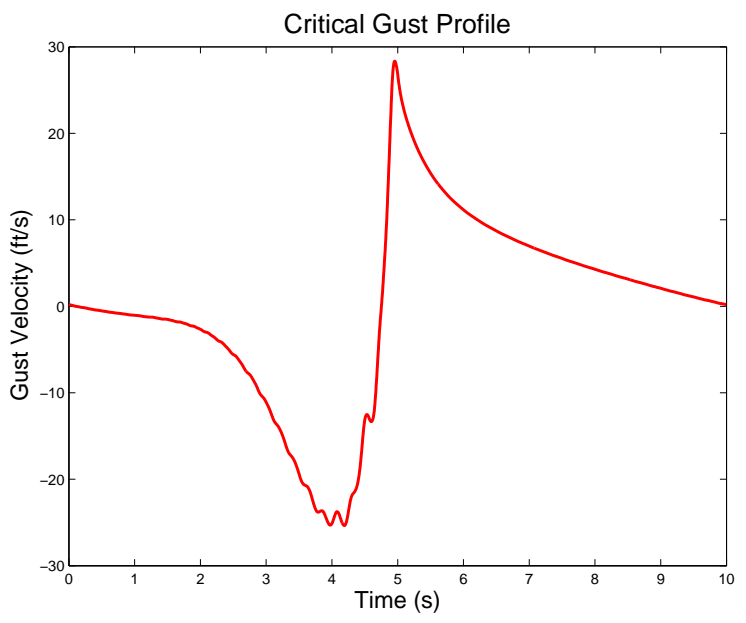

(a) Critical gust profile

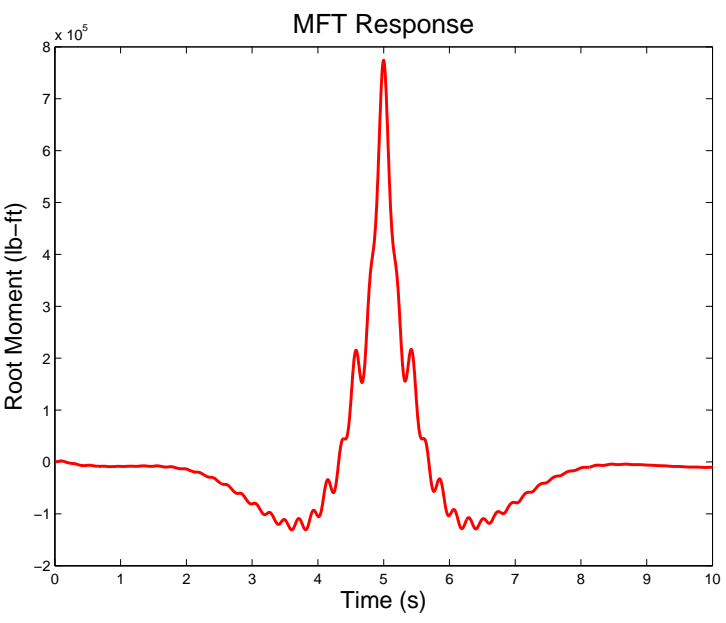

(b) Output response

Figure 12. Critical gust profile obtained from the critical excitation waveform, and the resulting root bending moment response. 


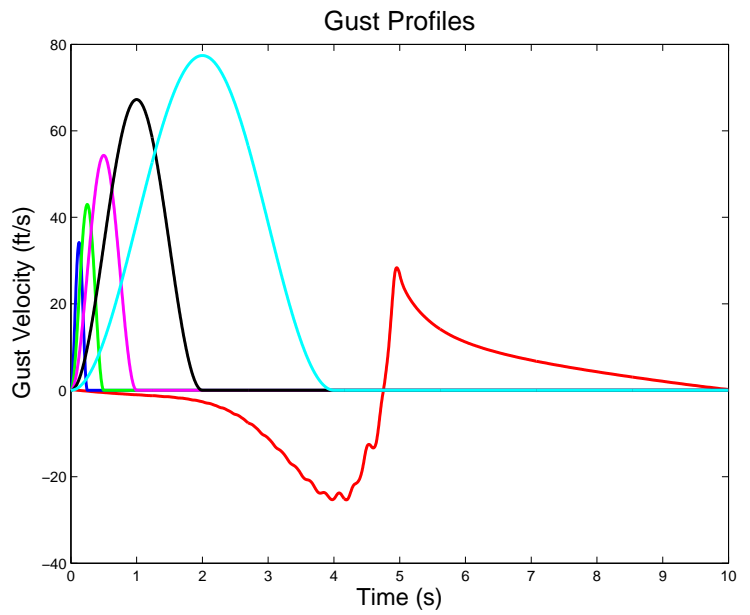

(a) Gust Profiles

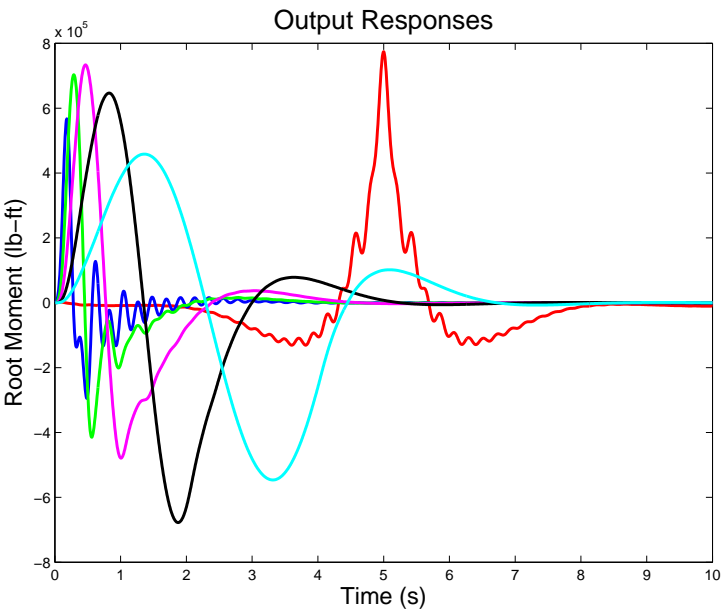

(b) Output Responses

Figure 13. Comparison of root bending moment output responses to the critical gust profile and 1-cos profiles of the same energy in excitation space.

\section{Conclusions and Ongoing Work}

We have presented several approaches for stochastic gust analysis and their application to a large dynamical system. The result of each analysis is a root-mean-square (RMS) value for one or more outputs of interest that can be used for design purposes as prescribed by the Federal Aviation Regulations. For a simple two degree of freedom aircraft model, all of these analysis approaches yielded the same RMS values, to numerical approximation errors. Two of the methods, power-spectral density integration and matchedfilter theory, were also applied to a large system obtained from a coupled aero-structural simulation. Again, similar RMS values were obtained from both approaches. An advantage of the matched filter theory analysis is that it yields a critical gust profile in addition to the RMS output value. We note that all of the methods are presented only for linear systems. Determining the effects of nonlinearities and extension to nonlinear systems will be the subject of future work.

Future work will also incorporate one or more of the stochastic gust analysis approaches into an aircraft design optimization setting. An important challenge in the optimization approach is the design of a gustload alleviation controller. This is not a trivial task, as many variables play a role in controller design, including the control architecture, sensor placement, actuation models, etc, and because much of this detail is traditionally not available in the conceptual design stage. In our ongoing work, we use a decomposition approach to formulating and solving the optimization problem, which permits use of specialized controller design techniques. In addition, future work will research the benefits of using multifidelity models to decrease the computational costs associated with the high fidelity analyses that may be necessary to capture the important physics.

\section{References}

\footnotetext{
${ }^{1}$ Kroo, I. M., "An Interactive System for Aircraft Design and Optimization," AIAA Paper 92-1190, 1992.

${ }^{2}$ Nissim, E., Caspi, A., and Lottati, I., "Application of the Aerodynamic Energy Concept to Flutter Suppression and Gust Alleviation by Use of Active Controls," NASA TN D-8212, 1976.

${ }^{3}$ Swaim, R. L., Schmidt, D. K., Roberts, P. A., and Hinsdale, A. J., "An Analytical Method for Ride Quality of Flexible Airplanes," AIAA Journal, Vol. 15, No. 1, 1977, pp. 4-7.

${ }^{4}$ Nam, C., Chattopadhyay, A., and Kim, Y., "Optimal Wing Planform Design for Aeroelastic Control," AIAA Journal, Vol. 38, No. 8, 2000, pp. 1465-1470.

${ }_{5}^{5}$ Aouf, N., Boulet, B., and Botez, R., "Robust Gust Load Alleviation for a Flexible Aircraft," Canadian Aeronautics and Space Journal (0008-2821), Vol. 46, No. 3, 2000, pp. 131-139.

${ }^{6}$ Kolonay, R., Eastep, F., and Sanders, B., "Optimal Scheduling of Control Surfaces on a Flexible Wing to Reduce Induced Drag," AIAA Paper 2004-4362, 2004.

${ }^{7}$ Thompson, E., Monsch, S., Camberos, J., and Eastep, F., "Induced Drag Minimization with Optimal Scheduling of Virtual Surface Deflection Boundary Conditions," AIAA Paper 2006-3160, 2006.

${ }^{8}$ Federal Aviation Regulations, "Part 25.341: Gust and Turbulence Loads," Federal Aviation Administration, 2008,
} 
http://www.faa.gov.

${ }^{9}$ Livne, E., "Integrated Aeroservoelastic Optimization: Status and Direction," Journal of Aircraft, Vol. 36, No. 1, 1999, pp. $122-145$.

${ }^{10}$ Zole, A. and Karpel, M., "Continuous Gust Response and Sensitivity Derivatives using State-Space Models," Journal of Aircraft, Vol. 31, No. 5, 1994, pp. 1212-1214.

${ }^{11}$ Livne, E., "Alternative Approximations for Integrated Control/Structure Aeroservoelastic Synthesis," AIAA Journal, Vol. 31, No. 6, 1993.

${ }^{12}$ Engelsen, F., Design-Oriented Gust Stress Constraints for Aeroservoelastic Design Synthesis, Ph.D. thesis, Department of Aeronautics and Astronautics, University of Washington, Seatte, WA, August, 2001.

${ }^{13}$ Zeiler, T. A. and Pototzky, A. S., "On the Relationship Between Matched Filter Theory as Applied to Gust Loads and Phased Design Loads Analysis," NASA CR 18102, 1989.

${ }^{14}$ Scott, R. C., Pototzky, A. S., and III, B. P., "Computation of Maximized Gust Loads for Nonlinear Aircraft Using Matched-Filter-Based Shcemes," Journal of Aircraft, Vol. 30, No. 5, 1993, pp. 763-768.

${ }^{15}$ Lee, Y.-N. and Lan, C. E., "Analysis of Random Gust Response with Nonlinear Unsteady Aerodynamics," AIAA Journal, Vol. 38, No. 8, 2000, pp. 1305-1312.

${ }^{16}$ Kanda, A. and Dowell, E. H., "Worst-Case Gust-Response Analysis for Typical Airfoil Section with Control Surface," Journal of Aircraft, Vol. 42, No. 3, 2005, pp. 956-962.

${ }^{17}$ III, B. P., Pototzky, A. S., and Woods, J. A., "NASA Investigation of a Claimed "Overlap" Between Two Gust Response Analysis Methods," Journal of Aircraft, Vol. 27, No. 7, 1990, pp. 606-611.

${ }^{18}$ Drela, M., "Integrated Simulation Model for Preliminary Aerodynamic, Structural, and Control-LAW Design of Aircraft," AIAA Paper 99-1394, 1999. 